\title{
Double-mode RR Lyrae models
}

\author{
G. Bono ${ }^{1}$, R.F. Stellingwerf ${ }^{2}$ \\ ${ }^{1}$ Trieste Astronomical Observatory, Italy ${ }^{2}$ Los Alamos National Laboratory, NM
}

\begin{abstract}
Double-mode RR Lyrae stars (RRd) represent a fundamental testing ground for the theory of stellar pulsation because they play an important role in understanding the interaction between different pulsation modes. In spite of the fact that from an observational point of view the identification of the fundamental (F) and first overtone (IO) periods needs only few periods of light curve coverage (Sandage et al. 1981, Nemec 1985), the theoretical models of doublemode and mixed-mode variables dating back to the original papers of Cox et al. (1983) and Stellingwerf (1975) were not integrated for long enough times to ensure proper mode identification. Recently Kovacs and Buchler (1992) using a standard radiative hydrodynamical code investigated the theoretical properties of RRd stars in more detail. This paper represents a brief description of the results of few cases computed adopting a non-linear, non-local treatment of convective transport, and long integration times to enable positive mode identification.
\end{abstract}

\section{Discussion}

The cases discussed, are model $2.3\left(\log L / L_{o}=1.8, T_{e}=7100 K, P_{0}=.5386 \mathrm{~d}, P_{1}=\right.$ $\left..4010 d, P_{1} / P_{0}=.7446\right)$ and model $4.6\left(\log L / L_{o}=1.5, T_{e}=6500 K, P_{0}=.4097 d, P_{1}=\right.$ $\left..3083 d, P_{1} / P_{0}=.7525\right)$. Both have been computed with $M=.65 M_{0}$ and King IA chemical composition (for mor details see Bono and Stellingwerf 1991, Stellingwerf and Bono 1992). The first one is located between the $I O$ and the $F$ blue edges, the latter one very close to the IO red edge. The period dispersion minimization technique (PDM, Stellingwerf 1978) has been applied to the radial variation of the surface zone for 20 consecutive periods to derive the amplitudes and periods of different modes. Along the run of models this analysis has been performed every 300 periods. It is worth emphasizing that this approach gives reliable results only if the physical and numerical approximations adopted in the code retain the exact energy conservation so that gains or losses from numerical perturbations a re avoided. In our case generally the energy conservation ranges between $10^{-8} \div 10^{-9}$ the initial value of the energy. Panel a of fig. 1 shows the switching from the $F$ to the $I O$ of model 2.3. The formula derived by Stellingwerf (1975b) and Cox et al. (1976) has been used to evaluate the switching time. For case 2.3 the switching rate ranges between .001 and $.0021 /(\mathrm{yr}$ $K)$, while the evolutionary time that a star with the same mass and almost the same chemical composition spends inside the instability strip is approximately $15000 \mathrm{yr} / \mathrm{K}$ 
(Castellani et al. 1991). Therefore a rough evaluation of the switching time is of the order of thousands of years $(5000 \pm 1000 \mathrm{yr})$. This value is more in agreement with the relatively large number of RRd stars found in galactic globular clusters and in the field. This value is an order of magnitude larger than the old estimation derived by Stellingwerf (1975). This discrepancy is essentially due to the decrease of the switching rate and partially to a small increase in the evolutionary time. Case 4.6 represents the first firm evidence of multimode stability in Pop. II variables. As a matter of fact the panels $b$ and $c$ of fig. 1 clearly show the stability of the first three modes obtained initiating the model in the $F$ mode with a surface amplitude of $10 \mathrm{~km} / \mathrm{s}$. To check the final amplitude and stability of these modes, the calculations have been repeated perturbing the IO linear radiative eigenfunctions with the same surface amplitude. Although, the amplitude of the IO remains constant over a long time, it is very comforting to note that the final amplitudes of the three modes are exactly the same. Unfortunately no firm conclusion can be reached concerning the location of RRd stars inside the stability strip and in particular about the physical mechanisms that govern the stability of these objects. Model 4.6 is located at the intersection between the resonance lines $\left(3 \nu_{0}=\nu_{1}+\nu_{2}\right)$ and $\left(4 \nu_{0}=3 \nu_{1}\right)$ therefore it will be necessary to extend the calculation along the resonance lines and the $F$ blue edge to account for the double-mode pulsation of RR Lyrae.
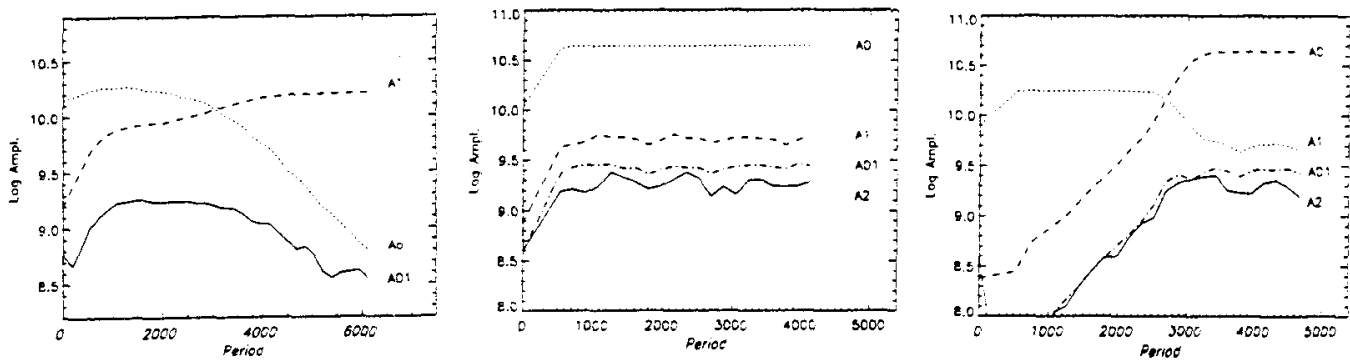

Figure 1 Radius amplitudes of cases 2.3 and 4.6. A0, A1, A2, A01 are the amplitudes of first three modes and the non-linear term.

\section{References:}

Bono, G., Stellingwerf, R.F., 1991, Mem. Soc. Astron. It., in press.

Castellani, V., Chieffi, A., Pulone, L., 1991, Ap. J. Suppl. 76, 911.

Cox, A.N., Hodson, S.W., Davey, W.L., 1976, In "Proceedings Solar and Stellar Pulsation Conference", ed. A.N. Cox, R.G. Dupree.

Cox, A.N., Hodson, S.W., Clancy, S.P., 1983, Ap. J. $266,94$.

Kovacs, G., and Buchler, R.J., 1992, Preprint.

Nemec, J.M., 1985, Astron. J. 90, 204.

Sandage, A., Katem, B., Sandage, M, 1981, Ap. J. Suppl. 46, 41.

Stellingwerf, R.F., 1975a, Ap. J. 195, 441.

Stellingwerf, R.F., 1975b, Ap. J. 199, 705.

Stellingwerf, R.F., 1978, Ap. J. 224, 953.

Stellingwerf, R.F., and Bono, G., 1992, This proceedings. 